\title{
Intraoperative Fluid Management in Combined Liver-Kidney Transplantation
}

\author{
Jong Hae Kim, M.D., Bo Reum Lim, M.D., and Jin Yong Jung, M.D.
}

\author{
Department of Anesthesiology and Pain Medicine, Catholic University of Daegu School of Medicine, Daegu, Korea
}

\begin{abstract}
A review of the literature regarding combined liver-kidney transplantation (CLKT) does not provide adequate central venous pressure (CVP) values that would allow for unimpaired hepatic venous outflow and early renal allograft diuresis during the procedure. We report a case of fluid management of CLKT based on the limited literature available in a 59-year-old male with liver cirrhosis and end-stage renal disease. During the preanhepatic phase, CVP was maintained at $5 \mathrm{mmHg}$. Following portal vein clamping, CVP was reduced to below $5 \mathrm{mmHg}$ until venovenous bypass was initiated. From the neohepatic phase to 1 hour before renal allograft reperfusion, CVP was slowly increased to $10 \mathrm{mmHg}$. Within an hour before renal allograft reperfusion, maximal crystalloid hydration was used to increase CVP to $15 \mathrm{mmHg}$. The urine output was replaced to maintain CVP at 8 to $10 \mathrm{mmHg}$ until the end of the surgery. The postoperative course was uneventful. In conclusion, fluid management tailored to each phase yielded beneficial results in a patient with CLKT.
\end{abstract}

Key Words: central venous pressure, kidney transplantation, liver transplantation.

The proportion of transplant recipients undergoing combined liver-kidney transplantation (CLKT) has increased since the model for end-stage liver disease system was introduced in the United States in February 2002. However, CLKT is still performed infrequently and constitutes only a very small fraction of abdominal transplantations. Thus, a review of the literature does not provide consistent answers about how to best optimize fluid management and whether to maintain a high or intentionally low central venous pressure (CVP) during CKLT because anesthesiologists have limited experience with this procedure. In particular, it is very challenging to maintain a CVP that is adequate to prevent impaired hepatic venous outflow and allograft congestion while simultaneously considering hydration for early diuresis of the renal allograft during the overlap of the neohepatic phase and the renal allograft pre-reperfusion phase. In addition, the most appropriate intravenous fluid that does not cause lactate, potassium, or chloride load to allografts during

Received on May 8, 2013, Revised on July 24, 2013,

Accepted on August 27, 2013

Correspondence to: Jin Yong Jung, Department of Anesthesiology and

Pain Medicine, Daegu Catholic University Medical Center,

33 Duryugongwon-ro 17-gil, Nam-gu, Daegu 705-718, Korea

Tel: +82-53-650-4505, Fax: +82-53-650-4517

E-mail: jychung@cu.ac.kr
CLKT is not known. However, there is some evidence regarding intraoperative fluid management for each phase [1-3] and the appropriate fluids [4,5] for use in single-organ transplantations. We report here on the intraoperative fluid management of CLKT based on this evidence [1-5] for a 59-year-old male diagnosed with hepatitis B related-liver cirrhosis and chronic renal failure caused by renal tuberculosis.

\section{CASE REPORT}

A 59-year-old Asian man $(168 \mathrm{~cm}, 71 \mathrm{~kg})$ with hepatitis B virus related-liver cirrhosis, end-stage renal disease caused by renal tuberculosis and hypertension presented to our center for CLKT. He was diagnosed with hepatitis B virus infection and renal tuberculosis 18 years ago. Fifteen years ago, he was discharged from our center after 1 month of admission for general weakness, during which he was diagnosed with hepatitis B related-liver cirrhosis and chronic renal failure caused by renal tuberculosis. The follow-up has since been lost. When he presented our center for CLKT, regular hemodialysis (3 times a week) began, and the patient was enrolled in the Korean Network for Organ Sharing. Forty-five days after enrollment, cadaveric hepatic and renal allografts became available. After the patient's informed consent was obtained, CLKT was planned.

Preoperative laboratory findings were as follows: leukocyte 
Table 1. Perioperative Laboratory Findings

\begin{tabular}{|c|c|c|c|c|c|c|}
\hline & Preoperative ${ }^{*}$ & Preanhepatic $^{\dagger}$ & Reperfusion $1^{\mp}$ & Neohepatic $^{8}$ & Reperfusion 2 & Postoperative \\
\hline $\mathrm{LC}\left(\right.$ cells $\left./ \mathrm{mm}^{3}\right)$ & 4,800 & 5,900 & 5,200 & 3,400 & 4,600 & 6,700 \\
\hline Hemoglobin (g/dl) & 6.2 & 5.8 & 5.3 & 7.0 & 8.4 & 10.0 \\
\hline Hematocrit (\%) & 17.3 & 16.2 & 15.0 & 20.5 & 24.2 & 28.1 \\
\hline $\mathrm{PC}\left(\right.$ cells $\left./ \mathrm{mm}^{3}\right)$ & 27,000 & 40,000 & 29,000 & 27,000 & 37,000 & 31,000 \\
\hline $\operatorname{AST}(\mathrm{IU} / \mathrm{L})$ & 51 & 36 & 274 & 508 & 746 & 862 \\
\hline ALT (IU/L) & 12 & 28 & 79 & 129 & 126 & 106 \\
\hline PT (sec) & 26.9 & 26.3 & 29.0 & 24.1 & 21.4 & 20.6 \\
\hline INR & 2.55 & 2.47 & 2.81 & 2.20 & 1.88 & 1.79 \\
\hline aPTT (sec) & $>180$ & 72.7 & $>180$ & $>180$ & 77.9 & 72.2 \\
\hline BUN (mg/dl) & 46.2 & 51.4 & 46.0 & 46.4 & 52.5 & 48.0 \\
\hline Creatinine $(\mathrm{mg} / \mathrm{dl})$ & 6.1 & 6.0 & 5.0 & 5.3 & 5.4 & 5.7 \\
\hline $\mathrm{Na}^{+}(\mathrm{mmol} / \mathrm{L})$ & 134 & 134 & 137 & 137 & 138 & 139 \\
\hline $\mathrm{K}^{+}(\mathrm{mmol} / \mathrm{L})$ & 3.7 & 4.0 & 3.7 & 3.2 & 3.0 & 3.2 \\
\hline $\mathrm{Cl}^{-}(\mathrm{mmol} / \mathrm{L})$ & 100 & 92 & 93 & 93 & 93 & 100 \\
\hline Serum glucose $(\mathrm{mg} / \mathrm{dl})$ & 101 & 106 & 179 & 216 & 216 & 157 \\
\hline Fibrinogen (mg/dl) & UA & 88 & 65 & 117 & 140 & 154 \\
\hline D-dimer $(\mu \mathrm{g} / \mathrm{ml})$ & UA & 11.77 & 7.07 & 6.34 & 17.5 & 9.16 \\
\hline $\mathrm{pH}$ & UA & 7.46 & 7.26 & 7.17 & 7.34 & 7.42 \\
\hline $\mathrm{PaO} 2(\mathrm{mmHg})$ & UA & 179 & 235 & 193 & 206 & 120 \\
\hline $\mathrm{PaCO} 2(\mathrm{mmHg})$ & UA & 32 & 37 & 41 & 37 & 44 \\
\hline Base excess & UA & -1.0 & -10.5 & -13.5 & -5.8 & 3.6 \\
\hline
\end{tabular}

${ }^{*} 24$ hours before surgery. ${ }^{\dagger} 1$ hour after surgical incision. ${ }^{\ddagger} 5$ minutes after hepatic allograft reperfusion. ${ }^{\S}$ Immediately after hepatic artery anastomosis. " 5 minutes after renal allograft reperfusion. "6 hours after end of the surgery. LC: leukocyte count; PC: platelet count; AST: aspartate aminotransferase; ALT: alanine aminotransferase; PT: prothrombin time; INR: international normalized ratio; aPTT: activated partial thromboplastin time; BUN: blood urea nitrogen; UA: unavailable.

count, 4,800 cells $/ \mathrm{mm}^{3}$; hemoglobin, $6.2 \mathrm{mg} / \mathrm{dl}$; hematocrit, $17.3 \%$; platelet count, $27,000 / \mathrm{mm}^{3}$; aspartate aminotransferase, $51 \mathrm{IU} / 1$; alanine aminotransferase, $12 \mathrm{IU} / 1$; blood urea nitrogen, $46.2 \mathrm{mg} / \mathrm{dl}$; creatinine, $6.1 \mathrm{mg} / \mathrm{dl} ; \mathrm{Na}^{+} 134, \mathrm{mmol} / \mathrm{l} ; \mathrm{K}^{+}, 3.7$ $\mathrm{mmol} / \mathrm{l} ; \mathrm{Cl}^{-}, 100 \mathrm{mmol} / \mathrm{l}$; prothrombin time, 26.9 seconds with international normalized ratio, 2.55; activated partial thromboplastin time, over 180 seconds; protein, $5.1 \mathrm{mg} / \mathrm{dl}$; and albumin, $3.3 \mathrm{mg} / \mathrm{dl}$ (Table 1). Hepatomegaly, splenomegaly and ascites were detected on physical examination of the abdomen. Preoperative posteroanterior chest X-ray and electrocardiogram were unremarkable.

Upon the patient's arrival at the operating theater, standard monitoring consisting of electrocardiogram, pulse oximetry, and noninvasive blood pressure was established. After intra-arterial catheterization for invasive arterial blood pressure monitoring, general anesthesia was induced using $250 \mathrm{mg}$ of intravenous thiopental and $0.25 \mathrm{mcg} / \mathrm{kg} / \mathrm{min}$ of intravenous remifentanil. Endotracheal intubation was facilitated with $50 \mathrm{mg}$ of intravenous rocuronium. The patient was mechanically ventilated with tidal volume of $10 \mathrm{ml} / \mathrm{kg}$, respiratory rate of 10 breaths/min, and $\mathrm{FiO}_{2}$ of 0.5 , which were adjusted to maintain arterial carbon dioxide and oxygen tension between 35-40 and 150-200 $\mathrm{mmHg}$, respectively. Anesthesia was maintained with desflurane and continuous infusion of intravenous remifentanil, and neuromuscular blockade was maintained with continuous infusion of rocuronium. After that, the right subclavian vein was cannulated using a triple lumen-central venous catheter for bolus administration, continuous infusion of intravenous medications, and continuous CVP monitoring. A Swan-Ganz catheter introducer was placed through the right internal jugular vein for continuous pulmonary artery pressure monitoring and massive volume replacement, and a double-lumen large-bore catheter for superior vena caval drainage of venovenous bypass was placed through right internal jugular vein. The right femoral artery and vein were cannulated for central arterial and infrahepatic inferior vena caval pressure monitoring, respectively.

After the anesthetic procedure and surgical draping were completed, the preanhepatic phase begun with the surgical incision of the abdomen. The evacuation of the ascites $(1,500 \mathrm{ml})$ from the peritoneal cavity reduced the CVP from $14 \mathrm{mmHg}$ to 8 $\mathrm{mmHg}$. Because of severe preoperative anemia, bleeding caused by liver dissection, and severe coagulopathy during the preanhepatic phase, blood products were transfused to recover the patient's oxygen-carrying capacity and to replace coagulation factors, and the CVP was maintained at approximately $5 \mathrm{mmHg}$ (Table 2). The more aggressive fluid restriction was im- 
Table 2. Perioperative Hemodynamic Parameters

\begin{tabular}{|c|c|c|c|c|c|c|}
\hline & $\begin{array}{c}\text { Preanesthetic } \\
\text { phase }^{*}\end{array}$ & $\begin{array}{c}\text { Preanhepatic } \\
\text { phase }^{\dagger}\end{array}$ & Anhepatic phase ${ }^{\ddagger}$ & Neohepatic phase ${ }^{\S}$ & $\begin{array}{c}\text { Renal allograft } \\
\text { pre-reperfusion phase }\end{array}$ & $\begin{array}{l}\text { Renal allograft } \\
\text { reperfusion phase }\end{array}$ \\
\hline SBP (mmHg) & 115 & $108(73,155)$ & $76(63,92)$ & $110(65,145)$ & $107(105,122)$ & $135(107,150)$ \\
\hline $\mathrm{DBP}(\mathrm{mmHg})$ & 63 & $47(33,65)$ & $38(33,45)$ & $50(28,62)$ & $50(47,57)$ & $65(55,73)$ \\
\hline HR (bpm) & 86 & $70(65,86)$ & $73(72,75)$ & $82(68,87)$ & $88(86,88)$ & $90(88,93)$ \\
\hline $\mathrm{CVP}(\mathrm{mmHg})^{* *}$ & UA & $4(0,7)$ & VVB & $8(3,11)$ & $13(11,16)$ & $11(8,12)$ \\
\hline PAOP (mmHg) & UA & $4(1,6)$ & VVB & $9(4,13)$ & $14(11,17)$ & $12(8,14)$ \\
\hline Cardiac Output (L/min) & UA & $6.2(3.3,7.9)$ & $4.4(2.1,4.8)$ & $7.0(2.2,8.8)$ & $8.1(7.7,9.8)$ & $7.7(7.0,8.5)$ \\
\hline $\mathrm{FVP}(\mathrm{mmHg})$ & UA & $10(8,13)$ & VVB & $9(3,12)$ & $\mathrm{UA}^{\dagger+}$ & $\mathrm{UA}^{\dagger+}$ \\
\hline Temperature $\left({ }^{\circ} \mathrm{C}\right)$ & UA & $36.4(36.3,36.6)$ & $35.9(35.4,36.3)$ & $36.4(36.3,36.5)$ & $36.1(36.0,36.2)$ & $36.3(36.2,36.4)$ \\
\hline
\end{tabular}

Values are expressed as median (range). ${ }^{*}$ Immediately before induction of anesthesia. ${ }^{\dagger}$ From surgical incision to excision of the native liver. ${ }^{\ddagger}$ From excision of the native liver to the hepatic allograft reperfusion. ${ }^{\S}$ From the hepatic allograft reperfusion to an hour before the renal allograft reperfusion. "During an hour before the renal allograft reperfusion. "from the renal allograft reperfusion to end of the surgery. ${ }^{* *} \mathrm{CVP}$ was targeted to $5,<5,10,15$, and, $8-10 \mathrm{mmHg}$ during preanhepatic, anhepatic, neohepatic (from total hepatectomy to portal vein cross-clamping), renal allograft pre-reperfusion phase, and renal allograft reperfusion phase, respectively. ${ }^{\dagger \dagger} \mathrm{FVP}$ is not available because the femoral vein catheter was removed not to impede the venous outflow of the renal allograft to the common iliac vein. SBP: systolic blood pressure; DBP: diastolic blood pressure; HR: heart rate; CVP: central venous pressure; PAOP: pulmonary artery occlusion pressure; FVP: femoral vein pressure; UA: unavailable; VVB: unreliable values due to venovenous bypass.

plemented to maintain the CVP at below $5 \mathrm{mmHg}$ following portal vein cross-clamping. The CVP value during venovenous bypass was not used as a target value during the anhepatic phase. From the neohepatic phase to 1 hour before renal allograft reperfusion, the CVP was slowly increased to $10 \mathrm{mmHg}$ (Table 2). Within an hour before renal allograft reperfusion, maximal crystalloid hydration was used to increase the CVP to $15 \mathrm{mmHg}$ (Table 2). Ten minutes after the reperfusion of the renal allograft, urine production was noted. The urine output was replaced to maintain the CVP value at approximately 8 to 10 $\mathrm{mmHg}$ until end of the surgery (Table 2). Inotropics and vasopressors were used to maintain mean arterial pressure at approximately $20 \%$ of the baseline, as appropriate. Plasmalyte was used to replace the fluid deficit and in combination with blood products. The serum potassium level was maintained within normal range throughout the surgery (Table 1). Prothrombin time and activated partial thromboplastin time improved after the reperfusion of the hepatic allograft (Table 1). Throughout the surgery, 2,340 $\mathrm{ml}$ of packed red blood cells, 2,160 $\mathrm{ml}$ of fresh frozen plasma, and 5,800 $\mathrm{ml}$ of Plasmalyte were infused, and the patient produced $2,000 \mathrm{ml}$ of urine output and lost 1800 $\mathrm{ml}$ of blood. After completion of the surgery, the patient was delivered to surgical intensive care unit and mechanically ventilated for 1 day. Two days after successful weaning from mechanical ventilation, the patient was transferred to the general ward. On the 8 th postoperative day, a reoperative laparotomy was performed to treat an intraperitoneal hematoma of unknown origin. Thereafter, the patient's postoperative course was uneventful, and he was discharged 38 days after the CLKT.

\section{DISCUSSION}

This case report showed that intraoperative fluid management tailored to each phase of CLKT produced favorable results. In particular, a slow increase of CVP with subsequent 1-hour maximal hydration immediately before renal allograft reperfusion seemed to prevent the impairment of hepatic venous outflow and allograft congestion, thus allowing hydration for early diuresis of the renal allograft during the overlap of the neohepatic phase and the renal allograft pre-reperfusion phase. In addition, Plasmalyte, which contains potassium, chloride, acetate, and gluconate, did not result in hyperkalemia, hyperchloremic metabolic acidosis, or allograft dysfunction.

A literature review revealed little evidence regarding intraoperative fluid management for CLKT. Faenza et al.[6] stated that the anesthetic approach to CLKT does not differ from the approach for isolated liver transplantation (LT), thus allowing for an accurate analysis of the filling values during the subsequent kidney transplantation (KT). In contrast, the anesthetic management of CLKT is identical to that described for single KT in Jankovic and Sri-Chandana's paper.[7] Although some authors have recently suggested a more detailed anesthetic procedure, they did not provide a clear explanation.[8]

Theoretically, maintaining a low CVP during the neohepatic phase may not impede blood outflow from the hepatic allograft and may potentially prevent the allograft dysfunction. However, high CVP should be maintained to optimize cardiac output and renal blood flow for the subsequent KT. To date, the precise CVP value that avoids hepatic allograft congestion and main- 
tains renal allograft blood flow at the same time during the neohepatic and renal allograft postreperfusion phase has not been clearly defined. Nevertheless, considering the results of Saner et al.'s study,[9] a CVP up to $10 \mathrm{mmHg}$ may be considered safe because no hepatic venous outflow impairment was observed at such levels. Moreover, maintaining CVP below $10 \mathrm{mmHg}$ during the neohepatic phase does not provide any benefit in terms of immediate postoperative allograft function, graft survival, or patient survival.[2] In a recent clinical trial, better intraoperative renal allograft turgidity, hemodynamic stability, earlier diuresis, and rapid improvement of postoperative renal allograft function were achieved with maximal hydration targeting a CVP of 15 $\mathrm{mmHg}$ within an hour before renal allograft reperfusion and subsequent replacement of allograft diuresis targeting a CVP of 8 to $10 \mathrm{mmHg}$.[3] Using these protocols, we increased the CVP to $10 \mathrm{mmHg}$ between the neohepatic phase and 1 hour before the renal allograft reperfusion. Within an hour before renal allograft reperfusion, the patient was maximally hydrated, with a target CVP of $15 \mathrm{mmHg}$. We then replaced the postvascularization urine output to maintain the CVP value at 8 to $10 \mathrm{mmHg}$ until end of the surgery.

A lower CVP facilitates venous drainage from the liver and may reduce bleeding during the preanhepatic phase. However, the accompanying renal compromise must be weighed against the benefit derived from lowering CVP. Massicotte et al.[10] suggested that lowering CVP to reduce blood transfusion during the preanhepatic phase did not increase the number of renal complications or the length of hospital stay. Moreover, they also found an improved 1-year survival rate for LT recipients.[10] Using this protocol, Feng et al.[1] achieved facilitated blood salvage with decreased secondary transfusion and liver function protection without a detrimental effect on renal function. In contrast, Schroeder et al.[11] found elevated creatinine levels, a more frequent need for dialysis and increased mortality in the low CVP group, in which the patients' CVP was maintained at $<$ $5 \mathrm{mmHg}$. Based on these results, the authors suggested that a low CVP should be avoided in LT. Thus, it is still controversial whether to maintain a low CVP during the preanhepatic phase. In the present case, we chose to adopt a low-CVP protocol because of the patient's already irreversible compromise of renal function. However, we could not find any reports relating to fluid management during venovenous bypass. Hence, fluid management was left to our discretion based on our clinical experiences.

Normal saline administration has been the standard of care for fluid management in patients undergoing KT on the theoretical basis that avoiding potassium loading prevents the develop- ment of intraoperative hyperkalemia in patients with end-stage renal failure.[12] However, the administration of large volumes of normal saline is associated with hyperchloremic metabolic acidosis, causing hyperkalemia through an extracellular shift of potassium ions.[13] The hyperchloremic metabolic acidosis that results from normal saline administration might also be detrimental to liver donors.[14] Lactate levels were increased in patients who received Ringer's lactate (containing $28 \mathrm{mmol} / \mathrm{L}$ of lactate) at the end of surgery in a study investigating the effect of normal saline, Ringer's lactate, and Plasmalyte on acid-base, potassium, and lactate levels during KT.[5] This study demonstrated the best metabolic profile (no significant increases in serum chloride, lactate, and potassium levels) in patients receiving Plasmalyte. When Plasmalyte was administered in living donors undergoing right hepatectomy, better lactate and liver profiles were shown compared to Ringer's lactate.[4] Magnesium (contained in Plasmalyte) preserves energy-dependent cellular activity and provides cellular protection during ischemic episodes.[15] Based on the results of the above studies, Plasmalyte, a balanced salt solution ( $\mathrm{pH} 7.4$ ) containing acetate and gluconate (instead of lactate) as bicarbonate precursors, 5 $\mathrm{mmol} / \mathrm{L}$ of potassium, a low chloride content $(98 \mathrm{mmol} / \mathrm{L})$, and $3 \mathrm{mmol} / \mathrm{L}$ of magnesium, was administered to maintain intravascular volume and in combination with blood products throughout the surgical period.

Recently, the idea that CVP reflects intravascular volume has been challenged.[16,17] The CVP is being replaced with the new hemodynamic parameter, such as stroke volume variation (SVV), which may be used as a surrogate to measure shifts in position of the Frank-Starling curve, for optimal fluid management in the patients receiving mechanical ventilation. Despite the superiority of SVV to CVP, the promising results of its use in the patients undergoing LT or KT are emerging [18,19] and there are no studies reporting the usefulness of SVV in CKLT at this point. However, some previous studies have reported CVP-targeting fluid management for LT or KT.[1-3] For this reason, we had to design the fluid management strategy using the "old" parameter (CVP) in the CKLT patient.

In conclusion, intraoperative fluid management tailored to each phase of CLKT surgery produced favorable results. However, evidence to support the protocol used in this case is lacking. Particularly, it is challenging for anesthesiologists to balance the intravascular volume to maintain the diuresis of the renal allograft and the venous outflow of the hepatic allograft after the neohepatic phase. Furthermore, it has not been determined whether Plasmalyte has a beneficial effect on the via- 
bility of hepatic and renal allografts in terms of avoiding lactate, potassium, and chloride loading in the patients undergoing CLKT. Therefore, further investigation is warranted to determine an intraoperative fluid management protocol tailored to CLKT.

\section{REFERENCES}

1) Feng ZY, Xu X, Zhu SM, Bein B, Zheng SS: Effects of low central venous pressure during preanhepatic phase on blood loss and liver and renal function in liver transplantation. World J Surg 2010; 34: 1864-73.

2) Cywinski JB, Mascha E, You J, Argalious M, Kapural L, Christiansen E, et al: Central venous pressure during the post-anhepatic phase is not associated with early postoperative outcomes following orthotopic liver transplantation. Minerva Anestesiol 2010; 76: 795-804.

3) Othman MM, Ismael AZ, Hammouda GE: The impact of timing of maximal crystalloid hydration on early graft function during kidney transplantation. Anesth Analg 2010; 110: 1440-6.

4) Shin WJ, Kim YK, Bang JY, Cho SK, Han SM, Hwang GS: Lactate and liver function tests after living donor right hepatectomy: A comparison of solutions with and without lactate. Acta Anaesthesiol Scand 2011; 55: 558-64.

5) Hadimioglu N, Saadawy I, Saglam T, Ertug Z, Dinckan A: The effect of different crystalloid solutions on acid-base balance and early kidney function after kidney transplantation. Anesth Analg 2008; 107: 264-9.

6) Faenza S, Arpesella G, Bernardi E, Faenza A, Pierucci E, Siniscalchi A, et al: Combined liver transplants: Main characteristics from the standpoint of anesthesia and support in intensive care. Transplant Proc 2006; 38: 1114-7.

7) Jankovic Z, Sri-Chandana C: Anaesthesia for renal transplant: Recent developments and recommendations. Curr Anaesth Crit Care 2008; 19: 247-53.

8) Ma Y, Wang GD, He XS, Li Q, Li JL, Zhu XF, et al: Simultaneous liver and kidney transplantation: Analysis of a single-center experience. Chin Med J (Engl) 2010; 123: 1259-63.
9) Saner FH, Olde Damink SW, Pavlaković G, van den Broek MA, Sotiropoulos GC, Radtke A, et al: Positive end-expiratory pressure induces liver congestion in living donor liver transplant patients: Myth or fact. Transplantation 2008; 85: 1863-6.

10) Massicotte L, Lenis S, Thibeault L, Sassine MP, Seal RF, Roy A: Effect of low central venous pressure and phlebotomy on blood product transfusion requirements during liver transplantations. Liver Transpl 2006; 12: 117-23.

11) Schroeder RA, Collins BH, Tuttle-Newhall E, Robertson K, Plotkin J, Johnson LB, et al: Intraoperative fluid management during orthotopic liver transplantation. J Cardiothorac Vasc Anesth 2004; 18: 438-41.

12) O'Malley CM, Frumento RJ, Bennett-Guerrero E: Intravenous fluid therapy in renal transplant recipients: Results of a US survey. Transplant Proc 2002; 34: 3142-5.

13) Halperin ML, Kamel KS: Potassium. Lancet 1998; 352: 135-40.

14) Chhibber A, Dziak J, Kolano J, Norton JR, Lustik S: Anesthesia care for adult live donor hepatectomy: Our experiences with 100 cases. Liver Transpl 2007; 13: 537-42.

15) Kim JE, Jeon JP, No HC, Choi JH, Lee SH, Ryu KH, et al: The effects of magnesium pretreatment on reperfusion injury during living donor liver transplantation. Korean J Anesthesiol 2011; 60: 408-15.

16) Wiesenack C, Fiegl C, Keyser A, Prasser C, Keyl C: Assessment of fluid responsiveness in mechanically ventilated cardiac surgical patients. Eur J Anaesthesiol 2005; 22: 658-65.

17) Marik PE, Baram M, Vahid B: Does central venous pressure predict fluid responsiveness? A systematic review of the literature and the tale of seven mares. Chest 2008; 134: 172-8.

18) Soga T, Kawahito S, Oi R, Kakuta N, Katayama T, Wakamatsu $\mathrm{N}$, et al: Recent less-invasive circulatory monitoring during renal transplantation. J Med Invest 2013; 60: 159-63.

19) Wang SC, Teng WN, Chang KY, Susan Mandell M, Ting CK, $\mathrm{Chu} \mathrm{YC}$, et al: Fluid management guided by stroke volume variation failed to decrease the incidence of acute kidney injury, 30-day mortality, and 1-year survival in living donor liver transplant recipients. J Chin Med Assoc 2012; 75: 654-9. 